Article

\title{
Expectation vs. Reality: Attitudes Towards a Socially Assistive Robot in Cardiac Rehabilitation
}

\author{
Jonathan A. Casas ${ }^{1}\left(\mathbb{D}\right.$, Nathalia Céspedes ${ }^{1}\left(\mathbb{D}\right.$, Carlos A. Cifuentes $\left.{ }^{1, *} \mathbb{(}\right)$, Luisa F. Gutierrez ${ }^{2}$, \\ Mónica Rincón-Roncancio ${ }^{2}$ (D) and Marcela Múnera ${ }^{1}$ (D) \\ 1 Biomedical Engineering Department, Colombian School of Engineering Julio Garavito, Bogotá 111166, \\ Colombia; jonathan.casas@escuelaing.edu.co (J.A.C.); nathalia.cespedes@mail.escuelaing.edu.co (N.C.); \\ marcela.munera@escuelaing.edu.co (M.M.); \\ 2 Fundación Cardioinfantil-Instituto de Cardiología, Bogotá 110131, Colombia; luisis81@yahoo.com (L.F.G.); \\ mrinron@hotmail.com (M.R.-R.) \\ * Correspondence: carlos.cifuentes@escuelaing.edu.co; Tel.: +57-031-668-3600
}

Received: 22 August 2019; Accepted: 22 October 2019; Published: 1 November 2019

\begin{abstract}
Currently, Social Assistive Robotics (SAR) is widely explored in different areas and scenarios. In cardiac rehabilitation, SAR has been recently implemented as a tool to improve the quality of the procedures and support patients to boost their performance. As cardiac rehabilitation comprises numerous sessions, such systems must guarantee to be effective in the long term. Therefore, to achieve this goal, it is important to understand how users, namely patients and clinicians who mostly know the needs and the therapy environment, perceive this technology. In this context, this paper presents the assessment of the attitudes towards a social robot in order to evaluate the expectation of potential new users, and perception of users who interacted with the social robot during a period of 18 weeks performing cardiac rehabilitation. A total of 43 participants (28 patients and 15 clinicians) were included in the study, and acceptance and perception factors were evaluated through a modified UTAUT questionnaire model and open discussion sessions. Results show that $75 \%$ of patients have positive thoughts regarding the usefulness, utility, safety, and trust perceived of a social robot, and $80 \%$ of clinicians consider that the robot is a useful tool for cardiac rehabilitation. Similarly, a more positive perception was noticed after the users interacted with the robot. Furthermore, this perception study allows the enhancement of the social model of interaction in the future, aiming to provide a more natural interaction trough personalized features, increasing social abilities and engagement of the users during the therapy.
\end{abstract}

Keywords: human-robot interaction; perception; social assistive robotics; robotic companion; UTAUT acceptance model; technology acceptance; cardiac rehabilitation

\section{Introduction}

The design and deployment of Socially Assistive Robotics (SAR) in healthcare and rehabilitation have increased in the last years [1]. Different areas have been explored from this robotics approach (e.g., stroke rehabilitation [2], autism [3], mental healthcare [4], elderly care [5], cardiac rehabilitation [6], among others). Most of these applications have shown promising results, where people feel encouraged and motivated to work along with robots in healthcare and rehabilitation environments [2,7]. However, most of these studies have been carried out in laboratory conditions with healthy patients or during short time interventions [8], which do not allow to have a complete perspective of the interaction between humans and robots, and it is often neglected several factors, such as novelty effect [9] and the adaptation of the technology [10]. In fact, only a few number of studies have explored long-term interactions with social robotic agents [11,12], where results report a considerable reduction in people's interest and motivation compared to the first interaction $[8,13,14]$. 
As SAR focuses on providing assistance through social interaction rather than physical interaction [15], as conventional robotics approaches do, these agents need to exhibit communicative and interactive skills that allow them to participate in human environments in a proper manner [16]. Therefore, to achieve that level of interaction, social robots require a set of technical features, such as: automated understanding of human behavior, quantitative diagnosis and assessment, sensor-based automated health data acquisition, and context-appropriate assistance that demand a high number of technological resources and complex developments [1]. Furthermore, as technology and robotic devices are exposed to technical failures, this situation can lead to slow response times, incorrect reactions, as well as low interactivity, which are factors that might be responsible of dropping the user's interest in the long-term interaction [8]. Based on this evidence, incorporating social agents into real in-clinic or in-home scenarios poses multiple challenges that researchers must overcome according to the user requirements and expectations.

This paper seeks to evaluate perception and attitudes towards a socially assistive robot designed to support the outpatient phase of cardiac rehabilitation therapies. In this scenario, patients are continuously monitored by a social robot (i.e., physiological, spatiotemporal, and exercise intensity parameters are considered) while performing physical activity on a treadmill [6]. Additionally, the robot performs behaviors (e.g., speech and body gestures) that potentially result in motivation and encouragement, aiming to increase patient engagement during sessions. The assessment will be carried out considering two main stakeholders: patients (direct robot users) and clinicians. To achieve this goal, a user perception and acceptance questionnaire, which is based on the adapted version of the Unified Theory of Acceptance and the Use of Technology (UTAUT) model [17] for social robots proposed in [18] was implemented in this work. This questionnaire was administered to a group of 20 patients without experience with the robot, eight patients who spent 18 weeks with the robot during therapy, and 15 clinicians who work on the cardiac rehabilitation service. The purpose of this work is to contrast initial attitudes and expectations against a post-interaction period, to understand how this technology can be more accepted by the users.

The remainder of this work is organized as follows. Section 2 describes the background as well as the context of this research, followed by Section 3, where the methodology and the experimental procedure carried out in the study is presented. In Section 4, results obtained from the perception questionnaire for both groups are described to be subsequently discussed in Section 5. Finally, this paper concludes by examining the hypothesis established according to the findings of the study.

\section{Background}

This section presents the background and context of the current research. First, the context of the cardiac rehabilitation scenario where the system was deployed is presented, followed by the description of the system's features and functionality. Additionally, this section presents models developed to estimate and assess user acceptance for technology, as well as techniques that have been adjusted to assess multiple dimensions on the perception of social assistive robots.

\subsection{Cardiac Rehabilitation Context}

Cardiovascular diseases (CVD) are considered one of the most critical causes of death worldwide. In 2017, it was reported that a total of 17.5 millions of deaths caused by CVDs, representing $31 \%$ of all deaths in the world [19]. Aiming to reduce this problematic, different clinics and rehabilitation centers provide cardiac rehabilitation $(\mathrm{CR})$ programs. These programs are designed to help people that have suffered a cardiovascular event to reduce the possibility of suffering a second event [20]. Conventional $\mathrm{CR}$ programs are structured in three phases (i.e., Phase I: inpatient; Phase II: outpatient; and Phase III: reinforcement) [21]. This research is focused on providing assistance to patients that completed the Phase I, and who are starting the Phase II or outpatient phase. This phase considers a twice-weekly program that takes an average of 18 weeks of physical exercise (treadmill-based physical activity), as well as an education program covering the prevention of risk factors and adoption of healthy habits, 
such as controlling blood pressure, cholesterol, weight, and stress management [22]. Prior to the intervention, an observation of the conventional therapy at the local clinic was performed. During this phase, the structure and stages of the therapy were identified. As illustrated in Figure 1, the session is divided in three stages: the first stage comprises the initial measurements and warming during the first 10 to $15 \mathrm{~min}$ of the session. The second stage is the exercise that is performed on a treadmill during 15 to $20 \mathrm{~min}$. Finally, after the physical activity, patients perform a 10 to $20 \mathrm{~min}$ cooldown together with the final measurements. Table 1 summarizes the measurements that are registered during each stage of the therapy. Pre-exercise and postexercise variables are meant to evaluate the patient's health condition before and after exercise (considering primarily their heart rate and blood pressure levels). During the exercise, the heart rate and intensity perception (i.e., Borg scale defined as a qualitative scale to estimate exertion during performing physical activity [23]), as well as the exercise conditions (speed and treadmill inclination) are considered.

\begin{tabular}{|c|c|c|c|c|}
\hline $\begin{array}{c}\text { Initial } \\
\text { measurements }\end{array}$ & Warning & Exercise & Cool-down & $\begin{array}{c}\text { Final } \\
\text { measurements }\end{array}$ \\
\hline
\end{tabular}

Figure 1. The therapy phases are illustrated: (1) Initial measurements and warming are performed during the first 10 to $15 \mathrm{~min}$ of the session. (2) The physical exercise is performed during 15 to $20 \mathrm{~min}$, and finally (3) the cooldown and final measurement are performed during the last 10 to $20 \mathrm{~min}$.

Table 1. Measured variables in cardiac rehabilitation (CR) session at the local clinic.

\begin{tabular}{ccc}
\hline & Variable & Unit \\
\hline \multirow{2}{*}{ Pre-Exercise Varibles } & Initial resting HR & $\mathrm{bpm}$ \\
& Weight & $\mathrm{kg}$ \\
& Initial blood pressure & $\mathrm{mmHg}$ \\
\hline \multirow{2}{*}{ Exercise Variables } & Speed & $\mathrm{mph}{ }^{*}$ \\
& Treadmill inclination & degrees \\
& Borg scale & \\
\hline \multirow{2}{*}{ Post-Exercise Variables } & Final HR & $\mathrm{bpm}$ \\
& Final blood pressure & $\mathrm{mmHg}$ \\
\hline \multicolumn{2}{c}{${ }^{*}$ treadmills provide speed in mph. }
\end{tabular}

Considering the important role that $\mathrm{CR}$ plays in the recovery and reduction of risk factors that could generate cardiovascular events, it is relevant to explore different approaches that can positively impact this service, improving program efficiency and quality. In this work, it is presented the development of socially assistive robot companions that can supervise training while motivating and keeping the patient engaged during the exercise. The research conducted by the authors was carried out in three stages: as illustrated in Figure 2a, an observation of conventional CR therapy was performed to identify relevant variables and parameters that the system should consider. Subsequently, a sensor interface, designed to measure multiple parameters (observed in the previous stage) during therapy, was integrated to a humanoid robot NAO (Softbank Robotics, France). This system was first evaluated under laboratory conditions with one healthy user (see Figure 2b) [20]. Finally, the system's performance was assessed under clinical conditions in a pilot study conducted with four patients during their first four therapy sessions (see Figure 2c), where the potential of the system was evidenced in [6].

This preliminary work has served to evaluate the reliability of the system, as well as the robustness in a real CR scenario. However, a long-term intervention assessment is required to identify users' perception and attitudes towards the social assistive robot. For this reason, this work focuses on evaluating these aspects on patients who finished the Phase II of the rehabilitation program and 
were exposed to social interaction with a robotic agent for 18 weeks. Furthermore, these results are contrasted with the perception and expectation that patients and clinicians had without having any previous interaction with the system. The next section describes the structure and functionality of the system.

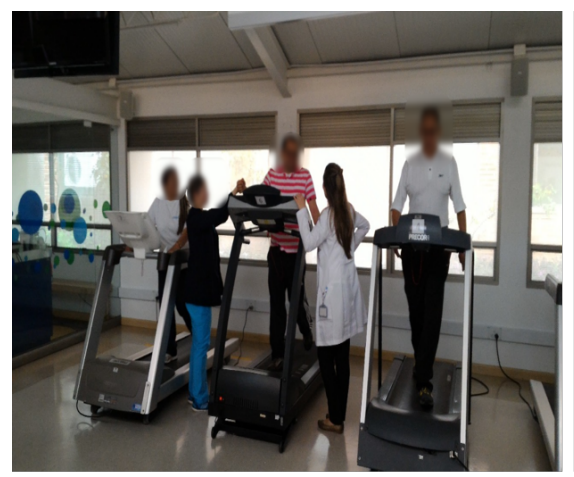

(a)

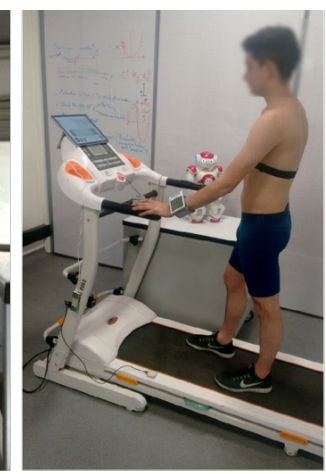

(b)

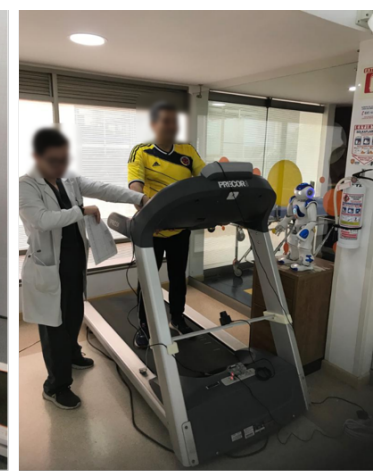

(c)

Figure 2. Social robotic companion in CR. (a) Exploration of the exercise session environment was carried out as a first stage. (b) The system design and performance were evaluated under laboratory conditions with a healthy user. (c) A pilot study was conducted with four patients under clinical conditions during the first four sessions.

\subsection{Robot Therapy in Cardiac Rehabilitation}

The robot therapy system is conformed by two main components. The first is the human-robot sensor interface which is designed to measure all therapy parameters that allow the social robotic agent to interact with the patient and the environment, and the second, a graphical user interface (GUI) that allows the patient to interact with the robot and receive the information processed by the sensor interface as the feedback of the training performance (see Figure 3). These components are detailed below.

\subsubsection{Human-Robot Sensor Interface}

The system is comprised of a sensor interface, aiming to measure all relevant variables of the therapy such as cardiopulmonary parameters (e.g., heart rate and blood pressure), spatiotemporal parameters (e.g., speed, cadence, and step length) and exertion perception scale (i.e., the Borg scale, defined as a qualitative scale to estimate exertion while performing physical activity [23]). To measure these variables a heart rate sensor (Zephyr HxM, Medtronic, USA), an IMU (MPU-6050, Invensense, USA) and a Laser Rangefinder (URG-04LX-UG01, Hokuyo, Japan) were integrated in the interface. Finally, a Nao robot (Softbank Robotics, France) is the social agent that manages all the data provided by the sensor interface to assist the patient and provide feedback, considering the events that occur within the session.

The architecture of the interface comprises different nodes (e.g., sensors and robot nodes) to properly handle the difference in sampling rates and the amount of information acquired by the system. In the case of the sensor interface, the output of each node is the data processed; and for the robot node is the corresponding behavior and feedback. Afterwards, a top layer of the interface architecture controls the time flow of the session (i.e., the start of the session, cooldown beginning and end of the session). Complete sensor data is stored in a database and is analyzed online to manage the robot interaction with the user. Robot's behaviors have been designed to interact with the patient while monitoring its performance, with the aim to having a positive influence on their motivation to perform the exercise (See Figure 3); and to communicate with the therapists if an event of emergency occurs during the therapy (e.g., heart rate over the maximum allowed level and dizziness). 
As depicted in Figure 3, the social robot based its behavior on three situations [6] (e.g., motivation, warning, and emergency, which are triggered depending on the time of the session and the data provided by the interface). Likewise, these behaviors were developed aiming to provide a similar assistance, as health professionals do. Previous observations of typical therapist-patient interactions were perform in order to develop suitable behaviors to each context. The custom-designed graphical user interface (GUI) is running on a tablet, which is available to allow the user to interact with the system, as well as to respond to the robot's requests. As it can be seen at the Figure 4, the GUI has different panels. The Control panel includes the widgets that are managed by the user (i.e., Borg Scale Buttons, Start, Stop, and Cooldown buttons), this widgets are in charge of record the time flow of the therapy and also store the Borg scale perceived by the patient through the session. Additionally, the Display panel (i.e., variables and patient displays) is developed to visualize the patients' parameters and data. This panel is useful for the clinicians as they are constantly watching over the heart rate status and the exercise parameters performed by the patients. Different behaviors require confirmation on the patient's side. Similarly, the robot asks for the Borg scale that must be delivered through the interface.

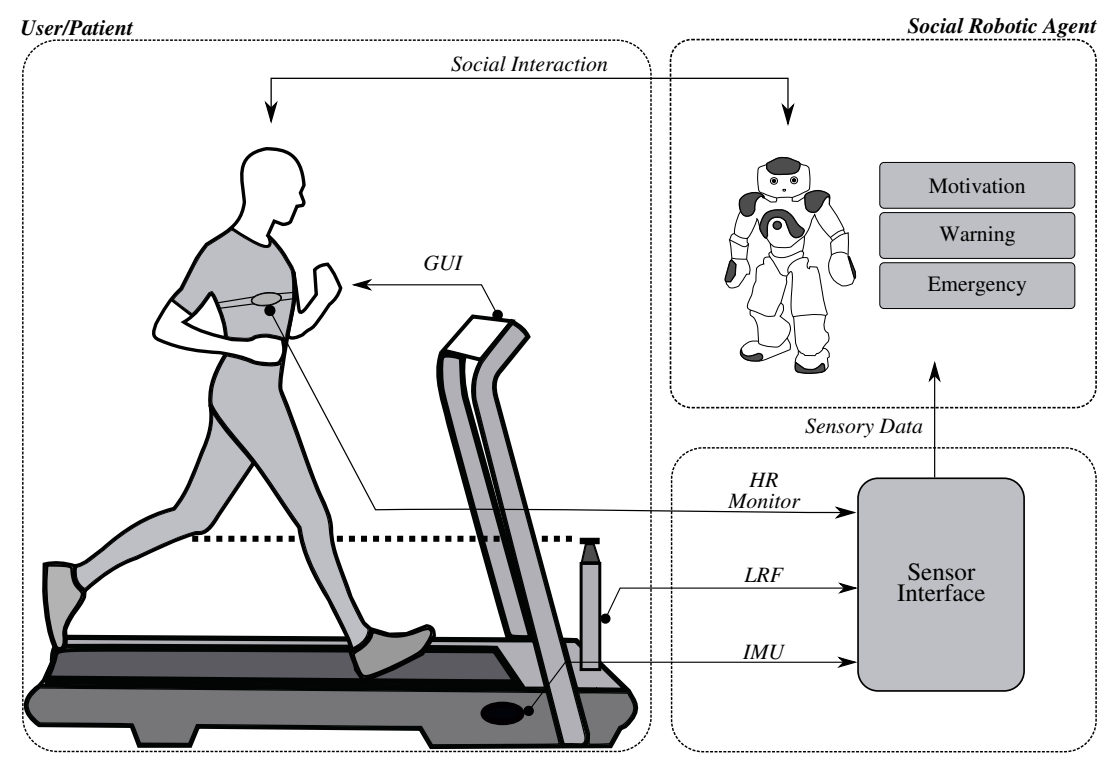

Figure 3. Experimental setup of a treadmill-based therapy at the cardiac rehabilitation facility.

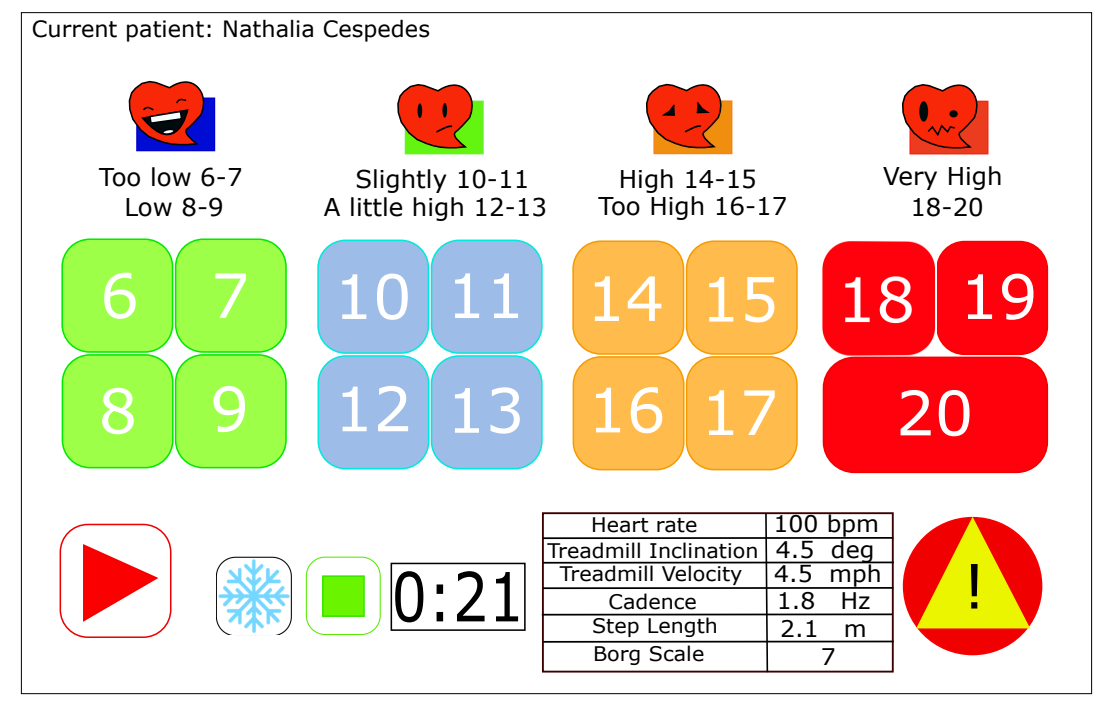

Figure 4. Graphical user interface (GUI) for cardiac rehabilitation human-robot interface. 


\subsubsection{Robot-Patient Interaction}

The interaction can be classified in three types: (1) There is a normal social interaction type, where the robot talks to the patient motivating and encouraging during the exercise, the robot uses its speech and body motions to express motivation and support. This interaction goes only in one direction, as the robot only talks to the patient without expecting any response. (2) The second type of interaction includes the GUI, where the robot performs a request that the patient must respond. In this case, the GUI enables a keyboard once the robot asked for this value. When the patient delivers the value, the keyboard is locked, and the GUI sends this information to the robot, which performs different behaviors considering the case (Table 2). Unlike the first mode of interaction, here there is a closed loop facilitated through the GUI having a bidirectional communication. Finally, (3) the third mode of interaction includes clinicians. In this case, the robot decides that clinicians must intervene in the therapy, where an alert has been detected due to an unexpected increase of the heart rate and the patient requires assistance by the clinicians to monitor their health condition. In this scenario, the patient is informed by the robot of the existence of an unusual condition and then clinicians are reported about the situation. The behaviors performed by the robot on each type of interaction are described in the Table 2.

These interactions can potentially improve cardiac rehabilitation results as the patients receive motivation constantly and they are monitored each session, unlike the conventional rehabilitation. The emergency state allows the therapist to control the session according the physiological parameters and also prevent a risk event. Finally, it is expected that the robot increases the adherence to the program, taking into account that motivational and cognitive aspects are fundamental to achieve a comprehensive rehabilitation $[24,25]$.

Table 2. Robot type of interactions.

\begin{tabular}{|c|c|c|}
\hline Type of Interaction & Behavior & Description \\
\hline $\begin{array}{l}\text { Patient-Robot } \\
\text { indirect interaction }\end{array}$ & $\begin{array}{l}\text { verbal and gestural } \\
\text { feedback }\end{array}$ & $\begin{array}{l}\text { The robot performs different poses to express the patient that } \\
\text { the work done is well. Phrases as "You are doing better today", } \\
\text { "Do not give up" and "You can do it", are randomly chosen } \\
\text { in order to positive influence the patient. }\end{array}$ \\
\hline $\begin{array}{l}\text { Patient-Robot } \\
\text { direct interaction }\end{array}$ & verbal feedback & $\begin{array}{l}\text { The patient registers the Borg scale (6-low exertion, to } 20-\text { high } \\
\text { exertion) and it is compared with the high rate. The robot } \\
\text { manages the following cases: } \\
\text { When there are a normal heart rate and the patient perceives } \\
\text { a low exertion, the robot says, "Thank you, it seems everything } \\
\text { is fine". When there is a high heart rate and the patient perceives } \\
\text { a high exertion, the robot uses the Therapist-Robot interaction type. } \\
\text { When there is a high heart rate and the patient perceives a low } \\
\text { exertion or the inverse way. The robot says: "Are you OK?, } \\
\text { please register again the Borg scale." If the heart rate continues } \\
\text { having high values the Therapist-Robot interaction is activated. }\end{array}$ \\
\hline $\begin{array}{l}\text { Therapist-Robot } \\
\text { interaction }\end{array}$ & $\begin{array}{l}\text { verbal and gestural } \\
\text { feedback }\end{array}$ & $\begin{array}{l}\text { The robot gives advice to the therapist when an emergency event } \\
\text { occurs. In the case of the Cardiac Rehabilitation program, when } \\
\text { the heart rate increases too much and exceeds the maximum heart } \\
\text { rate allowed (configured by the medical staff). The robot calls the } \\
\text { therapist shaking its hands and also uses verbal phrases such as: } \\
\text { "Doctor, the patient is feeling tired, can you come?" and "Doctor, } \\
\text { the patient needs help." The end of this behavior is triggered when } \\
\text { the doctor/therapist touches the robot head. }\end{array}$ \\
\hline
\end{tabular}

\subsection{User Acceptance Assessment}

Technology acceptance has been commonly described as the favorable reception and ongoing use of newly introduced devices and systems [26]. The Technology Acceptance Model (TAM) [27] has been adopted as one of the bases to evaluate acceptance in different applications (e.g., e-commerce acceptance 
model (EAM) to evaluate the technology acceptance associated to mobile health devices [28]). However, the TAM has been criticized as it lacks precision and ignores influential factors (e.g., complexity of the technology and user characteristics) that are relevant on many applications [26]. On response of this limitation, in [17], the Unified Theory of Acceptance and Use of Technology (UTAUT) was developed. This model has been successfully used in healthcare to evaluate different applications. For example, acceptance of web-based aftercare devices [29], therapist acceptance of new technology for rehabilitation [30], new models based on UTAUT for rehabilitation technologies [31], among others.

In the context of social robotic agents, different models have been used. For a physical robotic coach the Coach-Athlete Relationship Questionnaire (CART-Q) was used to evaluate relations between the robotic agent and the athlete [32]. Furthermore, in [33], the Godspeed questionnaire was implemented to assess different perceptions of the robot working as a trainer assistant. Similarly, the UTAUT has been also used to evaluate acceptance of social robots, such as the study presented in [34], where two scenarios were evaluated (i.e., an expressive robot personality vs. an introvert robot personality). With this model, the authors found that participants were more likely to accept the expressive personality over the introvert. Those evidences position the UTAUT model as a reliable basis to develop models that adjust better to the assessment of social robots.

However, people perceive social autonomous robots differently from other computer technologies due to the nature of the interaction (i.e., social robots seek to interact as humans do) [35]. Therefore, in some cases, conventional models of perception are limited, which have led researchers to develop adaptations of these models to meet their needs $[5,12,16,36]$. Heerink et al. implemented the UTAUT model, finding that the model had low explanatory power and it insufficiently indicated that social abilities contribute to the acceptance of a social robot [37]. In consequence, an adapted version was developed in [18], with the purpose of incorporating social aspects that are relevant to assess social robotic agents. They described user acceptance as "the demonstrable willingness within a user group to employ technology for the tasks it is designed to support". This model integrates several constructs that enables to have knowledge on the social factors influenced by a social robot (e.g., anxiety, attitude, facilitating conditions, social influence, intention to use, perceive adaptability, perceived enjoyment, perceived ease of use, perceived sociability, and perceived usefulness).

Moreover, in [38], the evaluation of a social robot was carried out based on the model proposed by Heerink et al. and in [5], the authors use the same model to measure the acceptance of a social humanoid robot by preschool and elementary school teachers. They conclude that participants have positive reactions and acceptance towards the robot, and the model also allows to evaluate other opinions of SAR that can significantly contribute to the development of this technology.

Other studies evaluate this approach in different kind of robots and scenarios (e.g., public spaces [39], virtual relational agents [40], elderly population [41], among others), showing that the modified UTAUT is a reliable method to asses social acceptance, to investigate users reactions. and analyze the societal impact.

Likewise, this research is oriented in the same direction, the acceptance assessment of the socially assistive robot to support cardiac rehabilitation therapies will be designed upon this model. The methodology conducted in this work is described in the next section.

\section{Materials and Methods}

As previously stated, this work seeks to evaluate the perceptions and attitudes of patients and clinicians towards a socially assistive robot incorporated in the phase II of the CR program. Aiming to achieve this goal, a questionnaire based on the model proposed by Heerink et al. [18] is conducted. This model seeks to evaluate acceptance of the robot as a cardiac therapy assistant in different dimensions (i.e., Utility / Advantages (U/A), Usefulness (U), Perceived Utility (PU), Safety (S), Ease of Use (EU), Perceived Trust (PT), Perceived Sociability (PS), and Social Presence (SP)). Each question was scored with a 5-point Likert scale (1: strongly disagree; 2: disagree; 3: neutral; 4: agree; 5: strongly agree). The study has been divided in two parts: The first, focuses on the perception and 
attitudes carried out with patients, and the second implements a focus group to analyze acceptance and perception of this technology with clinicians. Participants recruitment criteria and the protocol followed in this research is presented below.

\subsection{Participants}

A total of 43 participants performed the study, they were selected from a convenience sample (Table 3). For the patient group, 28 persons were recruited (male $=63.15 \%$, female $=36.84 \%$ in control group, male $=87.5 \%$, female $=12.5 \%$ in intervention, age $=54 \pm 8.48$ years old). These patients finished the inpatient phase and started the phase II or III of CR program. On the other hand, 15 clinicians who work in CR took part of the study (male $=6.66 \%$, female $=93.33 \%$, age $36.86 \pm 8.78$ years old, years of expertise years $11.13 \pm 7.68$ ). This group is conformed by different types of medical specialties (nursing, physiatry, occupational and physical therapy) and have no previous interaction with the robot, but they have at least a level of technical use with other technology devices as computers and tablets.

Table 3. Participants in the perception study.

\begin{tabular}{lll}
\hline & Study & Participants \\
\hline \multirow{2}{*}{ Patients } & Intervention: long-term study $(\mathrm{N}=8)$ & Patients attending Phase II \\
& Control: interviews $(\mathrm{N}=20)$ & Patients attending Phase II-III \\
\hline \multirow{2}{*}{ Clinicians } & \multirow{2}{*}{ Focus Group $(\mathrm{N}=15)$} & 3 Nurses \\
& & 4 Outpatient Clinic/CR \\
& & 6 Physiatrists \\
\end{tabular}

\subsection{Patients of the Study}

As illustrated in Table 3, for the patients group, two conditions were defined: (1) an intervention condition, where patients attending the phase II of the CR program participated in a long-term study. (2) An interview for a control group was conducted for patients attending phase II and III of CR. These groups are considered in order to compare opinions between patients that had a long-term interaction with the system and experienced the benefits and disadvantages (intervention group), and patients that have no experience with the robot (control group). Therefore, based on this comparison, it will be possible to analyze patients' perception and attitudes towards the incorporation of this technology in clinical applications, such as $C R$, from both perspectives. The description of the protocol implemented in both scenarios is presented below.

\subsubsection{Intervention Group}

The long-term study included patients that carried out therapies with the social assistive interface described before, during a period of time of 18 weeks ( 36 sessions of phase II). Once they finished the program, a perception questionnaire was applied to evaluate their attitudes towards the robot after a long-term interaction (Table A1). Additionally, two open questions, stated below, were implemented to have more detailed information about their experience and recommendations. As the open questions were formulated only in a positive manner, the results and discussion regarding these questions are subject to bias.

\section{Question 1:}

Would you recommend the use of the robotic system to the patients that are starting the rehabilitation therapy? 
Question 2:

According to your experience, what would you recommend to improve the robot-based therapy?

\subsubsection{Control Group}

As aforementioned, the interviews were applied to patients who are in an early stage of the phase II or phase III of the CR program. Similarly, they must have no previous experience with the above-described robotic system. According to these conditions, participants are briefly contextualized about SAR systems, the benefits that they can provide, and the variables that are measured in this application, followed by the presentation of a video where the real cardiac scenario is displayed and the robot with its functionality can be appreciated. Later, the questionnaire (Table A1) is introduced by the experimenter in order to specify the purpose of the questionnaire and the correct way to complete it. In addition to the questions based on Likert scale, two open questions were also included, regarding the questions of the users' opinions, which are stated below. As it was also mentioned in the intervention group, by the fact that the questions were formulated only in a positive manner, these questions are subject to bias.

Question 1:

Would you use the robot during the therapy? Why?

Question 2:

What expectations do you have about the therapy assisted by a robot?

\subsection{Clinicians of the Study}

A group of clinicians that work at the local clinic in areas associated to $\mathrm{CR}$ were invited to participate in a focus group at the clinic. The purpose of this activity was to understand how clinicians are familiarized with technology and the effects that this might have within rehabilitation programs. Furthermore, this focus group aimed to introduce SAR and discuss about their questions and concerns associated to the technology. The activities are carried out in order to identify how participants change their opinion and perception, once the robotic application has been explained and had the opportunity to witness an in situ demonstration. The structure of the focus group, described below, was inspired on the work developed in [42].

\section{Focus Group}

The schedule of this focus group is presented in Table 4. As performed with the patients group, an initial acceptance study based on the UTAUT [18] questionnaire was applied to clinicians to understand their perception regarding social robotics in CR (see Table A2), followed by a preliminary discussion session. In this section, current technologies used in medicine and social assistive robotics were explained to the medical staff in order to contextualize about the benefits (motivation, adherence and engagement) of using SAR in rehabilitation scenarios. Afterwards, a demonstration of the human-robot interface functionality was shown to the healthcare team. Finally, the healthcare team discussed new needs, challenges, modifications, and improvements that can be developed in the interface. 
Table 4. Focus group with clinicians.

\begin{tabular}{l}
\hline Pre-Session Questionnaire \\
\hline Acceptance \& usability based on UTAUT [18] \\
Opening Discussion \\
\hline Pre-Demo Discussion \\
\hline Introduction of conventional technologies in medicine \\
Presentation of Socially Assistive Robotics \\
Motivation: how social robots can benefit therapy programs \\
\hline Project Demonstration \\
\hline Demonstration of the cardiac rehabilitation SAR interface with Nao \\
\hline Post-Demo Discussion \\
\hline Demo feedback \\
Suggestions and applications with potential use
\end{tabular}

\section{Results}

The perception assessment for patients was completed by 28 participants (eight participated in the long-term study, and 20 in the interviews). Answers were grouped by category, to perform the analysis for each construct defined in the questionnaire. A Mann-Whitney-Wilcoxon (MWW) test was applied to determine significant differences on each construct between groups. This test is suitable for the five-point Likert scale, as it presents minimal type I error rates and equivalent power with the 2-sample $t$-test [43]. Furthermore, it has been demonstrated that the MWW test provides better results for small sample sizes than the $t$-test [44]. Results of this test are depicted in Table 5, where the $p$-value corresponding to each category was computed.

Table 5. Mann-Whitney-Wilcoxon test $p$-values.

\begin{tabular}{cc}
\hline Construct & Control vs. Intervention \\
\hline SP & 0.1612 \\
PS & 0.087 \\
PT & $\mathbf{0 . 0 0 0 0 6}$ \\
EU & $\mathbf{0 . 0 2 4 7 6}$ \\
S & 0.13782 \\
PU & $\mathbf{0 . 0 0 0 2 7}$ \\
U & $\mathbf{0 . 0 0 0 6 9}$ \\
\hline
\end{tabular}

As Table 5 shows, in most of the constructs defined in the questionnaire, a significant difference (i.e., $p$-value $<0.05$ ) was found. These categories are (Perceived Trust (PT), Ease of Use (EU), Perceived Utility (PU), and Usefulness (U)). On the other hand, Safety (S), Perceived Sociability (PS), and Social Presence (SP) do not present a significant difference between groups. The distribution of the Likert questions grouped by category is presented in Figure 5. Each category contains the results obtained from control and intervention groups. This plot is presented with a central axis, indicating a neutral position regarding the question (positive perceptions are plotted on the right side of the graph, while negative perceptions are represented on the left side).

Regarding the open questions, in the long-term study, all participants showed high interest in the robot-based therapy: $100 \%$ of the participants would recommend the therapy to incoming patients. On the other hand, in the interviewed group, $75 \%$ of participants found the therapy interesting and functional, and would recommend the system for future use, whereas $25 \%$ demonstrated no interest in the application and would not recommend the therapy due to different reasons that are considered and analyzed in the discussion section. 
For the clinicians, the UTAUT results are illustrated in Figure 6. Each construct represents a percentage of the total responses, taking into account the Likert scale. Although remarkable, the results regarding (U/A), (U), (PU), (PS), and (PT) categories have a positive score, and that of the (S) construct is negative, as that the question refers to the robot as a risk during $C R$ therapies.

Commentaries of the pre- and post-discussion, regarding clinicians' opinions on social robotics and the proposed interface, were recorded. Prediscussion results showed that clinicians were worried about being replaced by the robot, this was expressed in commentaries such as, "The robot can measure all the parameters that I usually monitor" and "The robot can replace my work." Also doubts in the functionality and features of the interface were expressed ("Why a robot? Can not be other device?" and "Sensors could fail in the measurements and report wrong data"). After the demo presentation and the introduction of social assistive robotics in healthcare (Table 4), these commentaries turn positive as a detailed explanation of the interface was given.

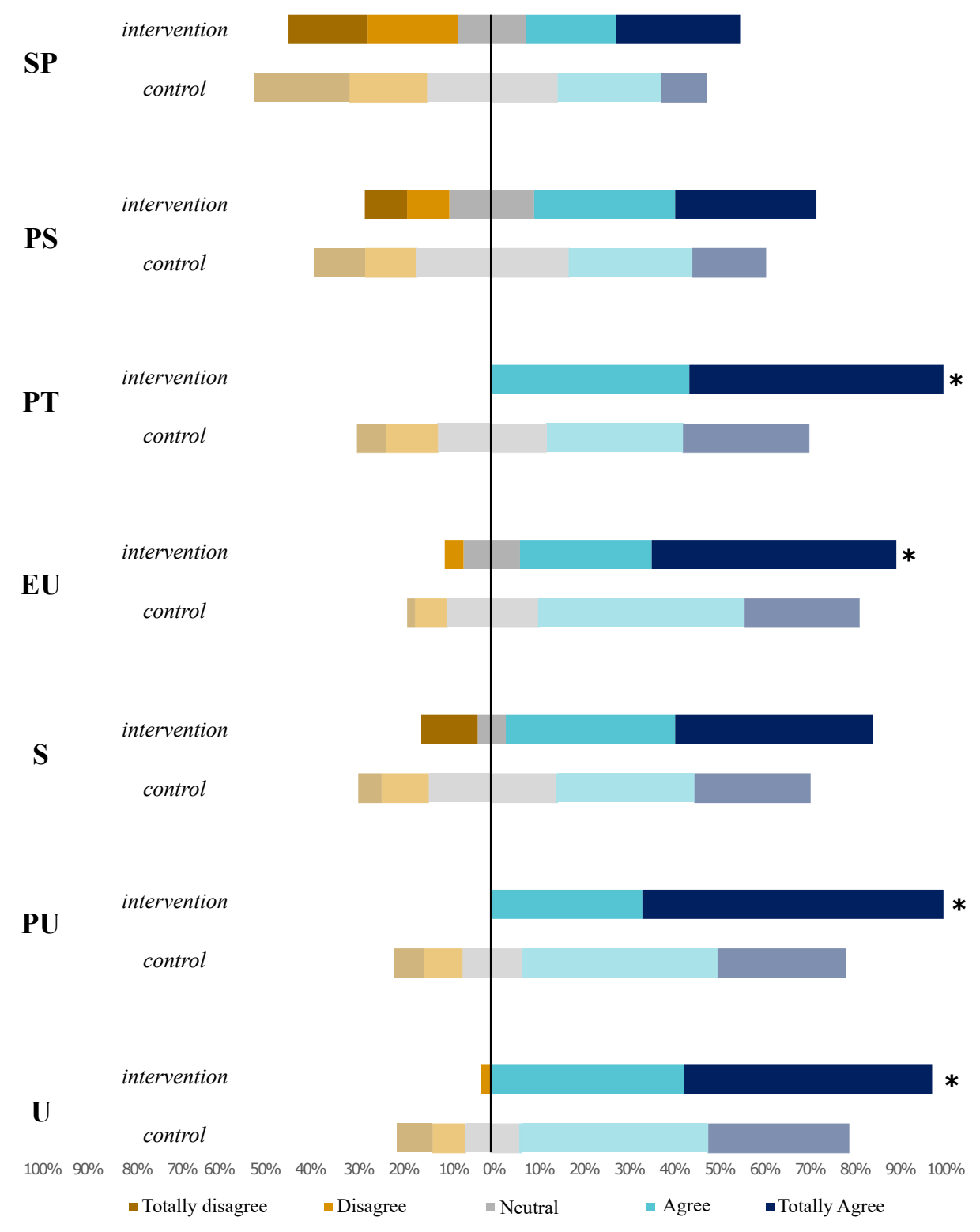

Figure 5. Likert scale distribution for each construct of the acceptance and perception questionnaire applied to patients. 


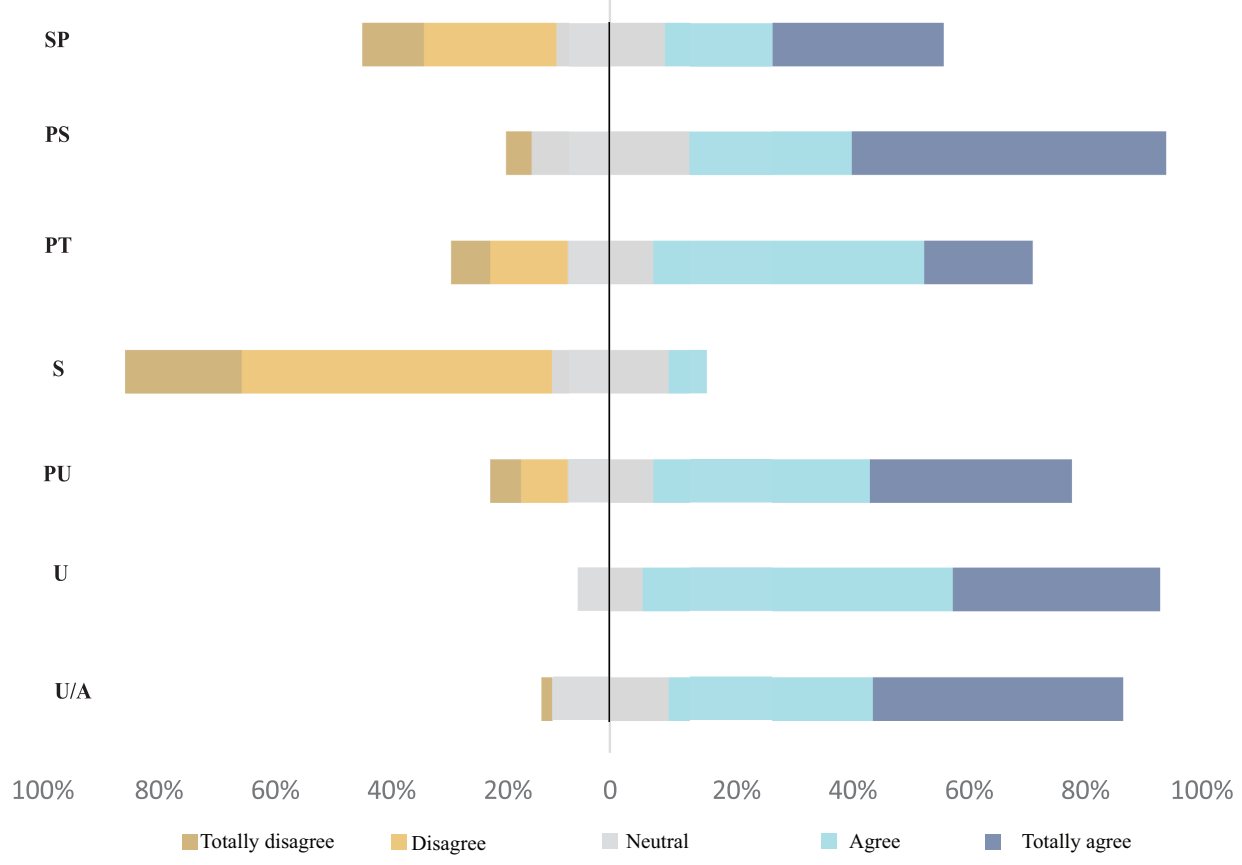

Figure 6. Likert scale distribution for each construct of the acceptance and perception questionnaire applied to therapists.

Results of the post-discussion showed an interest of the clinicians to improve and add features to the interface. Remarkable positive commentaries included the following, "Personally, I'm very interested in the capabilities of the robot to help me to measure some parameters so I can focus more on the other patients' needs", "It could be nice if the robot can be more sociable and less repetitive with its behaviors", "I'm very interested in knowing the performance of the patient at specific times, this feature should be added", "the online measurements that the robot provides are useful, this is important when a patient has an elevated heart rate" and "The use of robots can improve the techniques of cardiac rehabilitation. As the robot is constantly watching over patients' parameters, it is possible to perform high intensity training". These results are supported by $80 \%$ of the clinician group, who consider that using a robot during cardiac rehabilitation could be useful and adequate.

\section{Discussion}

This section addresses the results previously presented. The discussion will be focused first on the patient study, followed by the outcomes obtained with the clinicians study. This analysis will be carried out considering the result obtained on each construct of the perception's questionnaire.

\subsection{Patients}

Taking into account that this study considers a long-term interaction with a social assistive robot, several constructs were evaluated so as to know the opinion and the future requirements of the platform with the aim of improving the performance and interaction between users (patients and clinicians) and the robot.

For the patient group, an intervention and control condition were designed to compare the expectation and perception regarding the role of a social robot in cardiac rehabilitation. This was performed by means of a UTAUT questionnaire, showing remarkable results. As observed in Figure 5, the perceived trust is higher in the intervention group, than in the control group who expressed low confidence in the robot, where statements such as "not trustable" and "I would trust more in human therapists" were found. This is an expected reaction associated to the lack of experience and contact with the robot. However, not all patients expressed negative impressions towards the robot: there 
were found answers such as "I think is an appropriate approach", "I would be monitored all the time" or "It supports the patients' needs, as it contributes to the continuous and satisfactory improvement". This positive perception is endorsed by participants from the intervention group, who expressed a higher perceived trust to the robot. This indicator is important because the trust in the robot will influence the continuous use of the system in the future [12].

The utilitarian factor, which encloses ease of use (EU), perceived utility (PU), and usefulness (U), is fundamental for the engagement in long-term relationships [12]. Additionally, it has been demonstrated that users who did not perceive the robot as useful, stopped using it after the initial tests [45]. These categories showed differences between both conditions. For ease of use (EU), the intervention group perceives more ease of use than the control group. This is due to the time that these patients spent interacting with robot, where they have the opportunity to realize how complex the interaction with the robotic platform can be, and after a period of time, feel comfortable performing the therapy in presence of the social agent. As patients in the control group have a limited knowledge of the system and its functionality, it is difficult to understand the complexity of the use of the platform. Regarding the utility and the benefits that the system could provide in rehabilitation of patients, it was evidenced a higher perceived utility in the intervention group than in the control group. This result was expected, as patients that have the opportunity to interact with the robot during the complete rehabilitation process, can evidence the benefits of having the company of social agent during the treatment. During the therapies, they expressed to be motivated and encouraged to perform better ("I'm very encouraged to complete the rehabilitation", "The robot motivates me to exercise well" or "This is a novel tool that could help any kind of rehabilitation"). Although control patients perceive a high degree of utility, it can be evidenced that after the interaction, this expectation is overcame.

Finally, the usefulness construct is mostly focused on the perception that patients have of the system and its functionality (e.g., robot interventions, adaptability, interface, manipulation, etc.). In this case, the same pattern as the previous categories is presented. The intervention group attributes more usefulness to the system than the control group. Patients expressed: "I would like to have the opportunity to interact with the robot again" and that "the robot was beneficial to the development of the therapies". These results reflect the positive impact that the platform provided and the potential that it might have in future cardiac therapies.

Regarding the results of the utilitarian factor, it is possible to observe a mere-exposure effect for the intervention group, which refers to the tendency to evaluate the robot more positively after repeated interactions [46]. As mentioned before, the perception of the robot is better qualified for the group who interact with the robot more times as they familiarized with it. These results can be compared with studies presented in $[47,48]$, where researchers mentioned that the mere-exposure effect is a key factor to create more meaningful relationships with the robots.

Summarizing, the perception presented in both groups can be interpreted as positive, as Figure 5 shows most of the values located in the right side of the scale. However, it is also worth mentioning the results of the Social Presence (SP) category. In this case, there is a neutral opinion in both groups, which can mean that patients do not notice significant social features in the demonstration (control) and even after a considerable period of interaction (intervention). According to this result, it is necessary to improve this feature, as the impact and outcomes of the therapy can be potentially increased if the robot is more socially engaged to the patient [49]. Moreover, a higher perception of the robot sociability results in more intense social interactions [50], considering that most of the robot interventions are based on the social interaction and the way that it can develop in a social context. On the other hand, we observed that the intervention group does not recognize totally the robot as a real companion or that the robot was a friendly talker. This can be attributed to the feeling of shame when interacting with robots, as they are regarded as machines and devices [12]. These feelings are also identified in other studies where the researchers must previously introduce the robot and the possibility to have a social relationship with it, to improve the sociability perception $[13,51]$. 


\subsection{Clinicians}

For the clinician group the UTAUT shows positive opinions regarding (U/A), (U), (PU), (PT), and (PS) categories, which means that clinicians think that the robot and the parameters measured are useful and reliable in CR sessions. The (S) construct was scored negative due the question formulation; however, the results regarding this construct are positive as the clinicians do not consider the robot as a risk for the patients. Regarding perceive sociability (PS), the opinions in some questions were interesting, for example, for this construct in Q1 and Q5, clinicians think that the robot has to be only a coach with social cues and providing feedback the patient, but not a friendly companion as the patients needed to be concentrated in the therapy and the exercise. On the other hand, responses to Q3 showed that some clinicians think that the robot should not know the needs of the patients as that was the work of the medical staff. The social presence (SP) perception showed neutral response in general, this can be due to the perception of the robot as a social agent before the focus group was performed. In this case, the responses related to this construct showed that clinicians think that the robot could not have social skills as the humans (e.g., emotion and living creature) due the robot is perceived like an object.

One of the most important aspects that were observed during the focus group was the change of clinician perception as they went through the system's demonstration and received more information. As pointed out in the opening discussion, some clinicians perceived the incorporation of a social robot as a thread, as they regard the robot as a potential replacement. However, after the explanation of the technology and its objectives, it was emphasized that the robot must be considered as a tool that can improve their efficiency during therapy. Additionally, it was highlighted the relevance that the clinical staff has within the program. As a result of the informative talk, their conception of the system turned into a positive one, where clinicians showed interest and they also provided suggestions for improvements of the system. Additionally, more sociability capabilities were requested for robot behavior, as they realized the importance of this factor after the demonstrations and discussions; also, that the robot could provide statistics of the patients' performance as the system was capable to perform online monitoring during the complete outpatient phase. This feature can be interesting in order to support clinicians needs and help them with the diagnosis when patients finish phase II. Similarly, a more interactive robot model can be implemented using this information to provide better performance's feedback and interaction during therapy. Finally, comments made of the robot as a device to improve the cardiac rehabilitation process indicate that clinicians are interested to continue using the system.

Taking into account patient and clinician perception, one common factor that they emphasized is the sociability or social behaviors that the robot can exhibit. Results suggest that the current social features presented by the robot during therapy must be enhanced. This means that more algorithms and features, such as memory and vision recognition, can play an important role to increase the impact within therapies. However, there is a positive opinion regarding the usefulness of the system in this context, and the reliability of their monitoring that generates confidence to the users. These results are relevant to promote the adoption of the robot in CR and hold promising potential to continue investigating this approach.

\section{Conclusions and Future Work}

This work presented the assessment of the long-term perception and the attitudes that patients and clinicians had towards a socially assistive robot designed to support cardiac rehabilitation therapies. To achieve this goal, it was designed an experimental study for each group. In the case of patients, control (20 patients) and intervention (eight patients) conditions were evaluated, and for clinicians (15 persons), a focus group was performed with open discussion sessions, where opinions and comments where recorded. For both groups, participants were asked through a questionnaire, based on the modified UTAUT model presented by Heerink et al. [18], about their perception regarding different categories of the robot. 
Results show that patients present a lower expectation of the robot usefulness, sociability, and safety before interacting with it, and this expectation, in most cases, is overcame after a considerable time of interaction with the robotic platform. With the realization of this study, it was possible to demonstrate that although there is a bias when people consider to use this kind of technology, after they have the opportunity to familiarize with the robot and interact during a suitable period of time, their perception and attitudes towards the robot became more positive. Within the experimental study regarding the clinician group, positive responses to robot's usefulness, trust, and safety were found. In the same way as patients, before the demonstration of the interface, clinicians had doubts regarding the robots' role in cardiac rehabilitation, expressing concerns about being replaced and the data reliability. However, after the demo and the experience with the robot, patients manifested to be more motivated and engaged during the exercises; and clinicians showed a great interest on the interface giving recommendations and possible improvements that could be developed in a next version.

As was mentioned in the previous section, the social perception of the robot is not the desirable, patients (control and intervention), and clinicians, still have the same perception of the social cues of the robot. Therefore, the future work, that is proposed in this line, is the enhancement of the social model of interaction, aiming to provide a more natural interaction. For this purpose, it is meant to develop a personalized robot that is able to recognize each patient, monitoring their performance along the sessions and tracking the development of the complete rehabilitation phase. With this development, it is expected to increase the social abilities, and therefore the engagement of the users during the therapy.

Author Contributions: J.C. developed the cardiac interface and the UTAUT questionnaires. N.C. performed the questionnaires and the focus groups at the clinic. J.C. and N.C. led the manuscript writing. M.R. and L.G. reviewed the questionnaires and manage the experimental protocol. M.M. developed the experimental protocol and performed the statistics analysis for the data obtained. C.C. proposed and supervised the structure of the paper. M.M. and C.C. were involved in the revising and correcting the manuscript.

Funding: This work was supported in part by the Royal Academy of Engineering IAPP project Human-Robot Interaction Strategies for Rehabilitation based on Socially Assistive Robotics (grant IAPP $\backslash 1516 \backslash 137$ ), and Colciencias (grant 813-2017).

Acknowledgments: This work was carried out in collaboration with the Fundación Cardioinfantil-Instituto De Cardiología, where the experiments and protocols were performed. The authors acknowledge the work of Nathalie Zwickl for her support on the development of the focus group carried out with the clinicians.

Conflicts of Interest: The authors declare no conflicts of interest.

\section{Abbreviations}

The following abbreviations are used in this manuscript:

$\begin{array}{ll}\text { SAR } & \text { Socially Asstive Robotics } \\ \text { CR } & \text { Cardiac Rehabilitation } \\ \text { UTAUT } & \text { Unified Theory of Acceptance and Use of Technology } \\ \text { CVD } & \text { Cardiovascular Diseases } \\ \text { GUI } & \text { Graphical User Interface } \\ \text { EAM } & \text { e-commerce acceptance model } \\ \text { TAM } & \text { Technology Acceptance Model } \\ \text { CART-Q } & \text { Coach-Athlete Relationship Questionnaire } \\ \text { SP } & \text { Social Presence } \\ \text { PS } & \text { Perceived Sociability } \\ \text { PT } & \text { Perceived Trust } \\ \text { EU } & \text { Easy of Use } \\ \text { S } & \text { Safety } \\ \text { PU } & \text { Perceived Utility } \\ \text { U } & \text { Usefulness } \\ \text { U/A } & \text { Utility/Advantages }\end{array}$




\section{Appendix A. Patients' Perception Questionnaire}

Table A1. Perception Questionnaire for Patients.

\begin{tabular}{|c|c|c|}
\hline Construct & No. & Questions \\
\hline \multirow{5}{*}{$\mathrm{U}$} & 1 & I consider that using robots it's a good tool to assist cardiac rehabilitation therapies. \\
\hline & 2 & I consider that my interaction with the robot was comfortable. \\
\hline & 3 & I enjoyed when the robot gave me verbal encouragement when I did a good job. \\
\hline & 4 & I'm satisfied with the work that the robot did. \\
\hline & 5 & I consider that the robot adapts to my needs. \\
\hline \multirow{3}{*}{ PU } & 1 & I consider that the interaction with the robot was beneficial for my recovery. \\
\hline & 2 & I consider that the rol of the robot was important for the therapy development. \\
\hline & 3 & I think that the use of the robot helps me to compromise me to do a good job. \\
\hline \multirow{2}{*}{ S } & 1 & I feel safe at the therapies working with the robot. \\
\hline & 2 & I consider it was easy to give information to the robot. \\
\hline \multirow{3}{*}{ EU } & 1 & I consider that the robot is ease to use. \\
\hline & 2 & I consider that using the robot didn't affect the time of therapy sessions. \\
\hline & 3 & I consider that the robot's instructions were clear. \\
\hline \multirow{4}{*}{ PT } & 1 & The robot made me confident. \\
\hline & 2 & I did instruction the robot told me because I trusted him. \\
\hline & 3 & I like using the robot during the therapies. \\
\hline & 4 & It gave me confidence that the robot guides my therapy. \\
\hline \multirow{4}{*}{ PS } & 1 & I consider the robot a pleasant conversational partner. \\
\hline & 2 & I find the robot pleasant to interact with. \\
\hline & 3 & I feel the robot understands me. \\
\hline & 4 & I think the robot is nice. \\
\hline \multirow{5}{*}{ SP } & 1 & When interacting with the robot I felt like I'm talking to a real person. \\
\hline & 2 & It sometimes felt as if the robot was really looking at me. \\
\hline & 3 & I can imagine the robot to be a living creature. \\
\hline & 4 & I often think the robot is not a real person. \\
\hline & 5 & Sometimes the robot seems to have real feelings. \\
\hline
\end{tabular}

\section{Appendix B. Clinicians' Perception Questionnaire}

Table A2. Acceptance Questionnaire for Clinicians.

\begin{tabular}{ccl}
\hline Construct & No. & Questions \\
& 1 & I consider that using robots is a good tool to measure the HR and the BP during CR sessions. \\
& 2 & I consider that using robots it's a good tool to alert me if there is an abnormal heart rate. \\
& 3 & I consider that it can be useful know the number of steps made by a patient during a session. \\
U/A & 4 & I consider that using robots can help me carry out my tasks faster. \\
& 5 & I consider that the robot would not affect the time of cardiac rehabilitation sessions. \\
6 & I consider that using robots could improve my productivity during a therapy. \\
& 7 & I consider that the verbal motivation given by the robot could help the patient to be more productive. \\
& 1 & My interaction with the robot could be clear and understandable. \\
U & 2 & I might find the system easy to use. \\
& 3 & Learning to use the robot could be easy for me. \\
& 1 & I consider that using robots can bring benefits for the patients. \\
& 2 & I consider that using robots could help me to make a more personalized therapy for the patients. \\
& 3 & I consider that using robots could aid me to evaluate better the therapy. \\
& 4 & I consider that using robots could make my work more interesting. \\
& 5 & I feel that the robot could replace me.
\end{tabular}


Table A2. Cont.

\begin{tabular}{ccl}
\hline Construct & No. & Questions \\
\hline S & 1 & The robot would represent a risk to the patient's health. \\
& 1 & I would feel safe using the robot in the therapies. \\
PT & 3 & I could trust the work done by the robot in the sessions. \\
& 4 & I would like to use the robot during the therapies. \\
& 5 & I would trust the robot to help me guide the therapy. \\
& 1 & I consider that robots can be a pleasant conversationalist for the patient. \\
& 2 & I would like that the interaction between the patient and the robot can be pleasant. \\
PS & 3 & I would like the robot to understand the needs of the patient. \\
& 5 & I would like the robot to act as a friendly companion. \\
& 6 & I would like the robot to have a different modalities (monitoring, assistance and motivation). \\
& 1 & I consider that the interaction with the robot might feel like talking to a real person. \\
SP & 2 & I would consider good if the patient had the feeling that the robot will observe him in therapy. \\
& 3 & I consider it's good to imagine the robot as a living creature. \\
& 5 & I consider patients would usually think that the robot is not a real person. \\
& & I consider the robot could have real emotions. \\
\hline
\end{tabular}

\section{References}

1. Okamura, A.M.; Mataric, M.J.; Christensen, H.I. Medical and Health-Care Robotics. IEEE Robot. Autom. Mag. 2010, 17, 26-37. [CrossRef]

2. Matarić, M.J.; Eriksson, J.; Feil-Seifer, D.J.; Winstein, C.J. Socially assistive robotics for post-stroke rehabilitation. J. NeuroEng. Rehabil. 2007, 4, 5. [CrossRef] [PubMed]

3. Feil-Seifer, D.; Matarić, M.J. Toward Socially Assistive Robotics for Augmenting Interventions for Children with Autism Spectrum Disorders. In Experimental Robotics; Khatib, O., Kumar, V., Pappas, G.J., Eds.; Springer: Berlin/Heidelberg, Germany, 2009; pp. 201-210.

4. Rabbitt, S.M.; Kazdin, A.E.; Scassellati, B. Integrating socially assistive robotics into mental healthcare interventions: Applications and recommendations for expanded use. Clin. Psychol. Rev. 2015, 35, 35-46. [CrossRef] [PubMed]

5. Fridin, M.; Belokopytov, M. Acceptance of socially assistive humanoid robot by preschool and elementary school teachers. Comput. Hum. Behav. 2014, 33, 23-31. [CrossRef]

6. Casas, J.; Irfan, B.; Senft, E.; Gutiérrez, L.; Rincon-Roncancio, M.; Munera, M.; Belpaeme, T.; Cifuentes, C.A. Social Assistive Robot for Cardiac Rehabilitation: A Pilot Study with Patients with Angioplasty. In Proceedings of the Companion of the 2018 ACM/IEEE International Conference on Human-Robot Interaction, Chicago, IL, USA, 5-8 March 2018; ACM: New York, NY, USA, 2018; pp. 79-80. [CrossRef]

7. Gonzalez, J.C.; Pulido, J.C.; Fernandez, F.; Suarez-Mejias, C. Planning, execution and monitoring of physical rehabilitation therapies with a robotic architecture. Stud Health Technol. Inf. 2015, 210, 339-343.

8. Süssenbach, L.; Riether, N.; Schneider, S.; Berger, I.; Kummert, F.; Lütkebohle, I.; Pitsch, K. A robot as fitness companion: Towards an interactive action-based motivation model. In Proceedings of the 23rd IEEE International Symposium on Robot and Human Interactive Communication, Edinburgh, UK, 25-29 August 2014; pp. 286-293. [CrossRef]

9. Dc, W.; Gockley, R.; Bruce, A.; Forlizzi, J.; Michalowski, M.; Mundell, A.; Rosenthal, S.; Sellner, B.; Simmons, R.; Snipes, K.; et al. Designing Robots for Long-Term Social Interaction. In Proceedings of the 2005 IEEE/RSJ International Conference on Intelligent Robots and Systems, Edmonton, AB, Canada, 2-6 August 2005; pp. 2199-2204.

10. Riek, L.D. Healthcare Robotics. Commun. Acm 2017, 60, 68-78. [CrossRef]

11. Leite, I.; Martinho, C.; Paiva, A. Social Robots for Long-Term Interaction: A Survey. Int. J. Soc. Robot. 2013, 5, 291-308. [CrossRef]

12. de Graaf, M.M.; Allouch, S.B.; Klamer, T. Sharing a life with Harvey: Exploring the acceptance of and relationship-building with a social robot. Comput. Hum. Behav. 2015, 43, 1-14. [CrossRef] 
13. Kidd, C.D.; Breazeal, C. Robots at home: Understanding long-term human-robot interaction. In Proceedings of the 2008 IEEE/RSJ International Conference on Intelligent Robots and Systems, Nice, France, 22-26 September 2008; pp. 3230-3235. [CrossRef]

14. Kanda, T.; Hirano, T.; Eaton, D.; Ishiguro, H. Interactive Robots As Social Partners and Peer Tutors for Children: A Field Trial. Hum.-Comput. Interact. 2004, 19, 61-84. [CrossRef]

15. Feil-Seifer, D.; Matarić, M.J. Defining socially assistive robotics. In Proceedings of the 2005 IEEE 9th International Conference on Rehabilitation Robotics, Chicago, IL, USA, 28 June-1 July 2005; pp. 465-468. [CrossRef]

16. Shin, D.H.; Choo, H. Modeling the acceptance of socially interactive robotics: Social presence in human-robot interaction. Interact. Stud. 2011, 12, 430-460. [CrossRef]

17. Venkatesh, V.; Morris, M.G.; Davis, G.B.; Davis, F.D. User Acceptance of Information Technology: Toward a Unified View. MIS Q. 2003, 27, 425. [CrossRef]

18. Heerink, M.; Kröse, B.; Evers, V.; Wielinga, B. Assessing Acceptance of Assistive Social Agent Technology byOlder Adults: The Almere Model. Int. J. Soc. Robot. 2010, 2, 361-375. [CrossRef]

19. World Heart Organization. Cardiovascular Disease. 2017. Available online: https://www.who.int/en/ news-room/fact-sheets/detail/cardiovascular-diseases-(cvds) (accessed on 15 January 2019).

20. Lara, J.S.; Casas, J.; Aguirre, A.; Munera, M.; Rincon-Roncancio, M.; Irfan, B.; Senft, E.; Belpaeme, T.; Cifuentes, C.A. Human-robot sensor interface for cardiac rehabilitation. In Proceedings of the 2017 International Conference on Rehabilitation Robotics (ICORR), London, UK, 17-20 July 2017; pp. 1013-1018.

21. Graham, I.M.; Fallon, N.; Ingram, S.; Leong, T.; Gormley, J.; O’Doherty, V.; Maher, V.; Benson, S.E. Rehabilitation of the Patient with Coronary Heart Disease. In Hurst's The Heart, 13th ed.; Mc-Graw-Hill: New York, NY, USA, 2011; pp. 1-33.

22. Kraus, W.; Keteyian, S. Cardiac Rehabilitation; Humana Press: Totowa, NJ, USA, 2007.

23. Scherr, J.; Wolfarth, B.; Christle, J.W.; Pressler, A.; Wagenpfeil, S.; Halle, M. Associations between Borg's rating of perceived exertion and physiological measures of exercise intensity. Eur. J. Appl. Physiol. 2013, 113, 147-155. [CrossRef] [PubMed]

24. Siegert, R.J.; Taylor, W.J. Theoretical aspects of goal-setting and motivation in rehabilitation. Disabil. Rehabil. 2004, 26, 1-8. [CrossRef]

25. Maclean, N.; Pound, P. A critical review of the concept of patient motivation in the literature on physical rehabilitation. Soc. Sci. Med. 2000, 50, 495-506. [CrossRef]

26. Laver, K.; George, S.; Ratcliffe, J.; Crotty, M. Measuring technology self efficacy: Reliability and construct validity of a modified computer self efficacy scale in a clinical rehabilitation setting. Disabil. Rehabil. 2012, 34, 220-227. [CrossRef]

27. Davis, F.D.; Bagozzi, R.P.; Warshaw, P.R. User Acceptance of Computer Technology: A Comparison of Two Theoretical Models. Manag. Sci. 1989, 35, 982-1003. [CrossRef]

28. Schnall, R.; Higgins, T.; Brown, W.; Carballo-Dieguez, A.; Bakken, S. Trust, Perceived Risk, Perceived Ease of Use and Perceived Usefulness as Factors Related to mHealth Technology Use. Stud. Health Technol. Inform. 2015, 216, 467-471.

29. Hennemann, S.; Beutel, M.E.; Zwerenz, R. Drivers and Barriers to Acceptance of Web-Based Aftercare of Patients in Inpatient Routine Care: A Cross-Sectional Survey. J. Med. Internet Res. 2016, 18, e337. [CrossRef]

30. Liu, L.; Cruz, A.M.; Rincon, A.R.; Buttar, V.; Ranson, Q.; Goertzen, D. What factors determine therapists' acceptance of new technologies for rehabilitation-A study using the Unified Theory of Acceptance and Use of Technology (UTAUT). Disabil. Rehabil. 2015, 37, 447-455. [CrossRef]

31. Hatami Kaleshtari, M.; Ciobanu, I.; Lucian Seiciu, P.; Georgiana Marin, A.; Berteanu, M. Towards a Model of Rehabilitation Technology Acceptance and Usability. Int. J. Soc. Sci. Humanit. 2016, 6, 612-616. [CrossRef]

32. Griffiths, S.; Alpay, T.; Sutherland, A.; Kerzel, M.; Eppe, M.; Strahl, E.; Wermter, S. Exercise with Social Robots: Companion or Coach? In Proceedings of the Workshop on Personal Robots for Exercising and Coaching at the HRI 2018, Chicago, IL, USA, 5-8 March 2018.

33. Schneider, S.; Goerlich, M.; Kummert, F. A framework for designing socially assistive robot interactions. Cogn. Syst. Res. 2017, 43, 301-312. [CrossRef]

34. de Ruyter, B.; Aarts, E. Ambient Intelligence: Visualizing the Future. In Proceedings of the Working Conference on Advanced Visual Interfaces, Venezia, Italy, 23-26 May 2004; ACM: New York, NY, USA, 2004; pp. 203-208. [CrossRef] 
35. Weiss, A.; Bernhaupt, R.; Lankes, M.; Tscheligi, M. The USUS Evaluation Framework for Human-Robot Interaction. In Proceedings of the Symposium on New Frontiers in Human-Robot Interaction AISB2009, Edinburg, Scotland, 6-9 April 2009; pp. 158-165.

36. Heerink, M.; Kröse, B.; Wielinga, B.; Evers, V. Measuring the Influence of Social Abilities on Acceptance of an Interface Robot and a Screen Agent by Elderly Users. In Proceedings of the 23rd British HCI Group Annual Conference on People and Computers: Celebrating People and Technology, Cambridge, UK, 1-5 September 2009; British Computer Society: Swinton, UK, 2009; pp. 430-439.

37. Heerink, M.; Krose, B.; Evers, V.; Wielinga, B. Measuring acceptance of an assistive social robot: A suggested toolkit. In Proceedings of the 18th IEEE International Symposium on Robot and Human Interactive Communication, Toyama, Japan, 27 September-2 October 2009; pp. 528-533. [CrossRef]

38. Fink, J.; Lemaignan, S.; Dillenbourg, P.; Rétornaz, P.; Vaussard, F.C.; Berthoud, A.; Mondada, F.; Wille, F.; Franinovic, K. Which Robot Behavior Can Motivate Children to Tidy up Their Toys? Design and Evaluation of "Ranger". In Proceedings of the 2014 ACM/IEEE International Conference on Human-Robot Interaction, Bielefeld, Germany, 3-6 March 2014; pp. 439-446.

39. Weiss, A.; Bernhaupt, R.; Tscheligi, M.; Wollherr, D.; Kühnlenz, K.; Buss, M. A methodological variation for acceptance evaluation of human-robot interaction in public places. In Proceedings of the 17th IEEE International Symposium on Robot and Human Interactive Communication, Munich, Germany, 1-3 August 2008; pp. 713-718. [CrossRef]

40. Bickmore, T.; Schulman, D. Practical Approaches to Comforting. In Proceedings of the ACM CHI 2007: Conference on Human Factors in Computing Systems, San Jose, CA, USA, 30 April-3 May 2007; pp. 2291-2296.

41. Louie, W.Y.G.; McColl, D.; Nejat, G. Acceptance and attitudes toward a human-like socially assistive robot by older adults. Assist. Technol. 2014, 26, 140-150. [CrossRef] [PubMed]

42. Winkle, K.; Caleb-Solly, P.; Turton, A.; Bremner, P. Social Robots for Engagement in Rehabilitative Therapies. 2018; In Proceedings of the ACM HR1 2018: Conference on Human-Robot Interaction, Chicago, IL, USA, 5-8 March 2018; pp. 289-297. [CrossRef]

43. Joost, C.F.; Dodou, D. Five-Point Likert Items: T test versus Mann-Whitney-Wilcoxon. Pract. Assess. Res. Eval. 2010, 15, 1-16.

44. Blair, R.C.; Higgins, J.J. A Comparison of the Power of Wilcoxon's Rank-Sum Statistic to That of Student's t Statistic under Various Nonnormal Distributions. J. Educ. Stat. 1980, 5, 309. [CrossRef]

45. Fink, J.; Bauwens, V.; Kaplan, F.; Dillenbourg, P. Living with a Vacuum Cleaning Robot: A 6-month Ethnographic Study. Int. J. Soc. Robot. 2013, 5, 389-408. [CrossRef]

46. Zajonc, R.B. Attitudinal effects of "mere exposure": A reevaluation. J. Personal. Soc. Psychol. 1968, 9, 1-27. [CrossRef]

47. Kim, A.; Han, J.; Jung, Y.; Lee, K. The effects of familiarity and robot gesture on user acceptance of information. In Proceedings of the ACM/IEEE International Conference on Human-Robot Interaction, Tokyo, Japan, 3-6 March 2013; pp. 159-160. [CrossRef]

48. Jung, M.; Hinds, P. Robots in the Wild. ACM Trans. Hum.-Robot. Interact. 2018, 7, 1-5. [CrossRef]

49. Powers, A.; Kiesler, S.; Fussell, S.; Torrey, C. Comparing a computer agent with a humanoid robot. In Proceedings of the ACM/IEEE International Conference on Human-Robot Interaction, Arlington, VA, USA, 10-12 March 2007; ACM Press: New York, NY, USA, 2007; p. 145. [CrossRef]

50. Baddoura, R.; Venture, G. Social vs. Useful HRI: Experiencing the Familiar, Perceiving the Robot as a Sociable Partner and Responding to Its Actions. Int. J. Soc. Robot. 2013, 5, 529-547. [CrossRef]

51. Turkle, S. Alone Together: Why We Expect More from Technology and Less from Each Other, 1th ed.; Basic books: New York, NY, USA, 2011.

(C) 2019 by the authors. Licensee MDPI, Basel, Switzerland. This article is an open access article distributed under the terms and conditions of the Creative Commons Attribution (CC BY) license (http://creativecommons.org/licenses/by/4.0/). 